\title{
ARTICLE \\ Identification of compound D2923 as a novel anti-tumor agent targeting CSF1R
}

\author{
Ying-Qiang Liu ${ }^{1,2,3}$, Ya-Nan Wang ${ }^{2,3,4}$, Xiao-Yun Lu' ${ }^{5}$, Lin-Jiang Tong ${ }^{2}$, Yan Li ${ }^{2}$, Tao Zhang ${ }^{2,3,4}$, Qiu-Ju Xun ${ }^{3,5}$, Fang Feng ${ }^{2}$, Yu-Zhe Chen ${ }^{2,3}$, \\ Yi Su' ${ }^{2}$, Yan-Yan Shen ${ }^{2}$, Yi Chen ${ }^{2}$, Mei-Yu Geng ${ }^{2}$, Ke Ding ${ }^{5}$, Yan-Li Li ${ }^{1}$, Hua Xie ${ }^{2}$ and Jian Ding ${ }^{2}$
}

\begin{abstract}
Colony-stimulating factor 1 receptor (CSF1R) plays a critical role in promoting tumor progression in various types of tumors. Here, we identified D2923 as a novel and selective inhibitor of CSF1R and explored its antitumor activity both in vitro and in vivo. D2923 potently inhibited CSF1R in vitro kinase activity with an $\mathrm{IC}_{50}$ value of $0.3 \mathrm{nM}$. It exhibited 10- to 300 -fold less potency against a panel of kinases tested. D2923 markedly blocked CSF-1-induced activation of CSF1R and its downstream signaling transduction in THP-1 and RAW264.7 macrophages and thus inhibited the in vitro growth of macrophages. Moreover, D2923 dose-dependently attenuated the proliferation of a small panel of myeloid leukemia cells, mainly by arresting the cells at G1 phase as well as inducing apoptosis in the cells. The results of the in vivo experiments further demonstrated that D2923 displayed potent antitumor activity against M-NFS-60 xenografts, with tumor growth inhibition rates of $50 \%$ and $88 \%$ at doses of 40 and $80 \mathrm{mg} / \mathrm{kg}$, respectively. Additionally, D2923 was well tolerated with no significant body-weight loss observed in the treatment groups compared with the control. Furthermore, a western blot analysis and the immunohistochemistry results confirmed that the phosphorylation of CSF1R in tumor tissue was dramatically reduced after D2923 treatment, and this was accompanied by the depletion of macrophages in the tumor. Meanwhile, the expression of the proliferation marker Ki67 was also markedly decreased in the D2923 treatment group compared with the control group. Taken together, we identified D2923 as a novel and effective CSF1R inhibitor, which deserves further investigation.
\end{abstract}

Keywords: CSF-1R; small-molecule inhibitor; macrophage; myeloid leukemia

Acta Pharmacologica Sinica (2018) 39:1768-1776; https://doi.org/10.1038/s41401-018-0056-0

\section{INTRODUCTION}

Colony-stimulating factor 1 receptor (CSF1R, also known as CFMS) is a part of the class III growth factor receptor family that includes stem cell factor receptor (c-Kit), FMS-like tyrosine kinase 3 (Flt3), and platelet-derived growth factor receptor (PDGFR) $a$ and $\beta$ [1, 2]. CSF1R is activated by the homodimeric growth factors colony-stimulating factor-1 (CSF-1) and interleukin-34 (IL-34) and, subsequently, stimulates the proliferation and differentiation of monocyte/macrophage precursors [2-4]. CSF1R plays an important role in innate immunity by regulating the development of most tissue macrophages and osteoclasts, Langerhans cells of the skin, Paneth cells of the small intestine, and brain microglia [2]. It also regulates the differentiation of neural progenitor cells and controls the functions of oocytes and trophoblastic cells in the female reproductive tract $[2,5]$.

Accumulating evidence suggests the association of CSF1R with the development of different types of cancer [6, 7], including myeloid leukemia. Overexpression of CSF1R recruits macrophages into the tumor site [7] and induces them to transform into an M2like phenotype, which is generally called a tumor-associated macrophage (TAM) [8-10]. TAMs are reported to promote tumor growth, angiogenesis, invasion, and metastasis as well as resistance to therapy $[9,11,12]$. Currently, the inhibition of TAM survival/ activation by targeting CSF-1/CSF1R signaling has become a highly attractive strategy for cancer therapy [11,13-17]. There are several selective or nonselective small molecule CSF1R inhibitors reported that have advanced into different stages of clinical investigation [18-21]. PLX3397 (pexidartinib), an oral tyrosine kinase inhibitor of CSF1R, c-Kit, and mutant Flt3, is an agent with the broadest clinical development program and is undergoing phase III clinical studies in patients with diffuse type giant-cell tumors, which are highly dependent on CSF-1/CSF1R, and in patients with recurrent glioblastoma $[22,23]$. Though promising, the availability of the clinical data of PLX3397, on both safety and clinical activity, is still limited $[24,25]$. BLZ945 is a selective small molecule inhibitor of CSF1R, which is developed to a phase I/II clinical trial for the treatment of solid tumors [18, 26-28]. There is no selective CSF1R inhibitor approved by the U.S. Food and Drug Administration (FDA) for cancer treatment up to now. Therefore, it is desirable to develop novel CSF1R inhibitors for the treatment of cancers that are dependent on CSF-1/CSF1R.

\footnotetext{
${ }^{1}$ School of Life Science, Shanghai University, 200444 Shanghai, China; ${ }^{2}$ State Key Laboratory of Drug Research, Shanghai Institute of Materia Medica, Chinese Academy of Sciences, 201203 Shanghai, China; ${ }^{3}$ School of Pharmacy, University of Chinese Academy of Sciences, 100049 Beijing, China; ${ }^{4}$ School of Life Science and Technology, ShanghaiTech University, 201210 Shanghai, China and ${ }^{5}$ School of Pharmacy, Jinan University, No. 601 Huangpu Avenue, 510632 West Guangzhou, China

Correspondence: Yan-li Li (liyanli@shu.edu.cn) or Hua Xie (hxie@simm.ac.cn) or Jian Ding (jding@simm.ac.cn)

These authors contributed equally: Ying-Qiang Liu, Ya-Nan Wang.
}

Received: 12 April 2018 Revised: 29 May 2018 Accepted: 30 May 2018

Published online: 3 July 2018 
In the present study, we identified a novel CSF1R small molecular inhibitor, D2923. It potently inhibited the proliferation of macrophages induced by CSF-1 through CSF1R inhibition and downstream signaling suppression. It also showed potent antitumor activity against myeloid leukemia, which is highly dependent on CSF-1/CSF1R for growth, both in vitro and in vivo.

\section{MATERIALS AND METHODS}

Compound

(S)-3-(2,6-dichlorophenyl)-7-((3-methyl-4-(4-methylpiperazin-1-yl) phenyl)amino)-1-(1-propionylpyrrolidin-3-yl)-3,4-dihydropyrimido [4,5-d]pyrimidin-2(1H)-one (D2923). [a]20 D-25.000 (c 0.104, $\mathrm{MeOH}) .{ }^{1} \mathrm{H}$ NMR $\left(400 \mathrm{MHz}_{1} \mathrm{CDCl}_{3}\right) \delta 8.03(\mathrm{~s}, 0.6 \mathrm{H}), 8.01(\mathrm{~s}, 0.4 \mathrm{H})$, 7.44-7.42 (m, 2H), 7.37-7.29 (m, 3H), $7.10(\mathrm{~s}, 0.6 \mathrm{H}), 7.02-7.00$ $(\mathrm{m}, 1.4 \mathrm{H}), 5.59-5.57(\mathrm{~m}, 1 \mathrm{H}), 4.53-4.52(\mathrm{~m}, 2 \mathrm{H}), 4.04-3.91(\mathrm{~m}, 2 \mathrm{H})$, 3.76-3.72 $(\mathrm{m}, 1 \mathrm{H}), 3.50-3.42(\mathrm{~m}, 1 \mathrm{H}), 2.93-2.91(\mathrm{~m}, 4 \mathrm{H}), 2.75-2.73$ $(\mathrm{m}, 1 \mathrm{H}), 2.58-2.57(\mathrm{~m}, 4 \mathrm{H}), 2.36(\mathrm{~s}, 3 \mathrm{H}), 2.30(\mathrm{~s}, 3 \mathrm{H}), 2.27-2.25$ $(\mathrm{m}, 2 \mathrm{H}), 2.23-2.22(\mathrm{~m}, 1 \mathrm{H}), 1.14(\mathrm{t}, J=8.0 \mathrm{~Hz}, 3 \mathrm{H}) .{ }^{13} \mathrm{C} \mathrm{NMR}(125$ $\left.\mathrm{MHz}_{1} \mathrm{CDCl}_{3}\right) \delta 172.7,172.4,159.5,157.6,157.3,153.9,153.8,151.7$, $147.5,136.7,135.4,135.2,135.1,134.4,134.3,133.6,130.0,129.9$, $128.9,123.2,123.1,119.7,118.6,118.5,102.7,55.8,52.8,52.1,52.0$, 51.1, 47.4, 46.3, 45.8, 45.6, 44.6, 29.0, 28.0, 27.4, 26.7, 18.1, 18.0, 9.2. HRMS (ESI) for $\mathrm{C}_{31} \mathrm{H}_{36} \mathrm{CIN}_{8} \mathrm{O}_{2}\left[\mathrm{M}^{+} \mathrm{H}\right]^{+}$, calcd: 623.2411. Found: 623.2410. HPLC analysis: $\mathrm{MeOH}-\mathrm{H}_{2} \mathrm{O}$ (85:15), $12.01 \mathrm{~min}, 99.58 \%$ purity. D2923 was dissolved in dimethyl sulfoxide at $10 \mathrm{mM}$ and, subsequently, was serially diluted to specific concentrations.

\section{Kinase assays}

The ability of D2923 to inhibit the activity of a panel of kinases was tested using an enzyme-linked immunosorbent assay (ELISA). The kinase enzymes CSF1R, c-Kit, PDGFRa, PDGFR $\beta$, ErbB2, Trk-A, VEGFR1, Flt-3, IR, Trk-B, Trk-C, TEC, JAK3, EPH-A2, JAK1, JAK2, and RON were purchased from Eurofins (Brussels, Belgium). A total of $20 \mu \mathrm{M}$ Poly (Glu, Tyr) $4: 1$ (Sigma, St Louis, MO, USA) was precoated in 96-well ELISA plates as a substrate. Active kinases were incubated with the indicated drugs in $1 \times$ reaction buffer $(50 \mathrm{mM}$ HEPES pH 7.4, $20 \mathrm{mM} \mathrm{MgCl}_{2}, 0.1 \mathrm{mM} \mathrm{MnCl}_{2}, 0.2 \mathrm{mM} \mathrm{Na}_{3} \mathrm{VO}_{4}, 1 \mathrm{mM}$ DTT) containing $5 \mu \mathrm{M}$ ATP at $37^{\circ} \mathrm{C}$ for $1 \mathrm{~h}$. After the incubation, the wells were washed with PBS and were then incubated with an anti-phosphotyrosine (PY99) antibody (Santa Cruz Biotechnology, Santa Cruz, CA) followed by an incubation with a horseradish peroxidase-conjugated secondary antibody. The wells were visualized using o-phenylenediamine (OPD), and the absorbance was read with a multiwall spectrophotometer (VERSAmax, Molecular Devices, Sunnyvale, (A, USA) at $490 \mathrm{~nm}$.

Cell lines and cell culture

The mouse myelogenous leukemia cell line M-NFS-60 (RPMI1640+ $0.05 \mathrm{mM}$ 2-mercaptoethanol $+62 \mathrm{ng} / \mathrm{mL}$ CSF-1 + 10\%FBS), human peripheral blood monocyte THP-1 cells (RPMI1640 + 10\% FBS + $0.05 \mathrm{mM}$ 2-mercaptoethanol), RAW264.7 mouse peritoneal macrophages (DMEM $+10 \% \mathrm{FBS}$ ), the human acute myelogenous leukemia cell line KG-1 (IMDM $+20 \% \mathrm{FBS})$, the human acute lymphoblastic leukemia cell line RS4;11 (RPMI1640 + 10\%FBS) and the human erythrocyte leukemia cell line HEL (RPMI1640 + glucose + sodium pyruvate $+10 \% \mathrm{FBS}$ ) were purchased from the American Type Culture Collection (ATCC, Manassas, VA, USA). The mouse colon cell line MC38 (DMEM + 10\%FBS) was purchased from the FuDan IBS Cell Center (FDCC, Shanghai, China). Mouse brain glioma DBT cells (RPMI1640 + 10\%FBS) were purchased from the Ministry of Health Pharmaceutical Biological Products Appraisal Institute (Beijing, China). All the cell lines were maintained in $5 \% \mathrm{CO}_{2}$ at $37^{\circ} \mathrm{C}$ with a humidified atmosphere.

Western blot analysis

RAW264.7 cells were seeded into six-well plates at $2 \times 10^{6}$ cells per well. The cells were washed three times with PBS after adherence to remove serum protein, and then, the cells were starved in serum-free medium for $12 \mathrm{~h}$. For the THP- 1 or M-NFS- 60 cell lines, the cells were directly seeded into six-well plates at $2 \times 10^{7}$ cells per well with serumfree medium for $12 \mathrm{~h}$. Different concentrations of D2923 were added to the wells, and the cells were then incubated for $2 \mathrm{~h}$ before stimulation by $100 \mathrm{ng} / \mathrm{mL}$ CSF-1 per well or $2 \mathrm{~mL}$ of fresh MC38 or DBT CM, which was cultivated for 2 days. The cells were washed three times, and whole-cell lysates, for western blotting, were extracted with RIPA buffer ( $25 \mathrm{mM}$ Tris-HCl pH 7.6, $150 \mathrm{mM} \mathrm{NaCl}, 1 \% \mathrm{NP}-40,1 \%$ sodium deoxycholate, $0.1 \%$ SDS, protease, and phosphatase inhibitor cocktail). The cell lysates $(30 \mu \mathrm{g})$ were loaded onto an SDS-PAGE gel and were transferred to a polyvinylidene fluoride membrane. The anticipated objective strap position on the membrane was clipped, and the membrane was incubated overnight at $4{ }^{\circ} \mathrm{C}$ with the antibodies p-CSF1R(\#3155S), CSF1R(\#3152S), p-AKT (\#4060S), AKT (\#9272S), and ACTIN(\#3700S), which were purchased from Cell Signaling Technologies (Cambridge, MA, USA). Recombinant human CSF-1 (C417) and recombinant mouse CSF-1 (CB34) were purchased from Novoprotein (Shanghai, China). A western blot analysis was subsequently performed with standard procedures.

\section{Proliferation assays}

RAW264.7 cells were plated into 24-well plates with $5 \times 10^{3}$ cells per well. Then, the cells were treated with $1 \mu \mathrm{M}$ D2923, $1 \mu \mathrm{M}$ PLX3397 or vehicle control, in the presence or absence of CSF-1 (CB34, Novoprotein, Shanghai, China) at a concentration of $100 \mathrm{ng} / \mathrm{mL}$. For all the experiments, the compounds and cytokines were renewed every $24 \mathrm{~h}$. The cell numbers were recorded every $6 \mathrm{~h}$ using IncuCyte Zoom. Compared with the Raw264.7 cells, the THP-1 cells grew mainly in a state of suspension such that there was some difference between them. Briefly, the THP-1 cells were plated in 24-well plates with $5 \times 10^{3}$ cells per well. After adding drugs and cytokines, the cell numbers were recorded by a Beckman Coulter (Z2, CA, USA).

\section{Tumor cell viability assays}

Tumor cell viability was evaluated using the CCK-8 Cell Counting Kit (A311-01/02, Vazyme, Nanjing, China). The cells were seeded into 96-well plates at 3000 cells per well and were cultured overnight to allow for recuperating. Then, they were treated with different concentrations of D2923 and were incubated for $72 \mathrm{~h}$. The CCK-8 solution $(10 \mu \mathrm{L})$ was added to each well after $72 \mathrm{~h}$. Then, the plate was distributed and incubated for a certain time at $37^{\circ} \mathrm{C}$ in an incubator (depending on the type of cell, the was usually 1-4 h). The absorbance was measured at $450 \mathrm{~nm}$ using a microplate reader. A calibration curve was prepared using the data obtained from the wells that contained known numbers of viable cells.

\section{Flow cytometry analysis}

Cell-cycle determination. M-NFS-60 cells were collected and dispersed into six-well plates with $2 \times 10^{5}$ cells/well. Different concentrations of D2923 were used to treat the cells, and then, they were incubated for $48 \mathrm{~h}$. The cells were collected and fixed with $70 \%$ ethanol overnight after washing twice with PBS. The cells were resuspended in $500 \mu \mathrm{L}$ of PBS, containing $50 \mu \mathrm{g} / \mathrm{mL}$ propidium iodide $(\mathrm{PI})$ and $100 \mu \mathrm{g} / \mathrm{mL}$ RNase $\mathrm{A}$, for $30 \mathrm{~min}$ at $4{ }^{\circ} \mathrm{C}$ away from light. The flow cytometry analysis was performed using a BD Aria III Flow Cytometer (BD Biosciences, San Jose, California, USA), and the data were analyzed using Modfit LT 4.0 software.

Cell apoptosis examination. Apoptosis was investigated using the Annexin V-FITC Apoptosis Detection Kit (A211-01/02 Vazyme, Nanjing, China). M-NFS-60 cells were seeded into a six-well plate and were incubated in gradient concentrations of D2923 for $48 \mathrm{~h}$. The cells were washed twice with PBS and were resuspended in $500 \mu \mathrm{L}$ of PBS containing $5 \mu \mathrm{L}$ of Annexin V-FITC and $5 \mu \mathrm{L}$ of the PI Staining Solution, for $30 \mathrm{~min}$. The flow cytometry analysis was performed using a BD Aria III Flow Cytometer (BD Biosciences, San Jose, California, USA), and the data were analyzed by FlowJoVX software. 

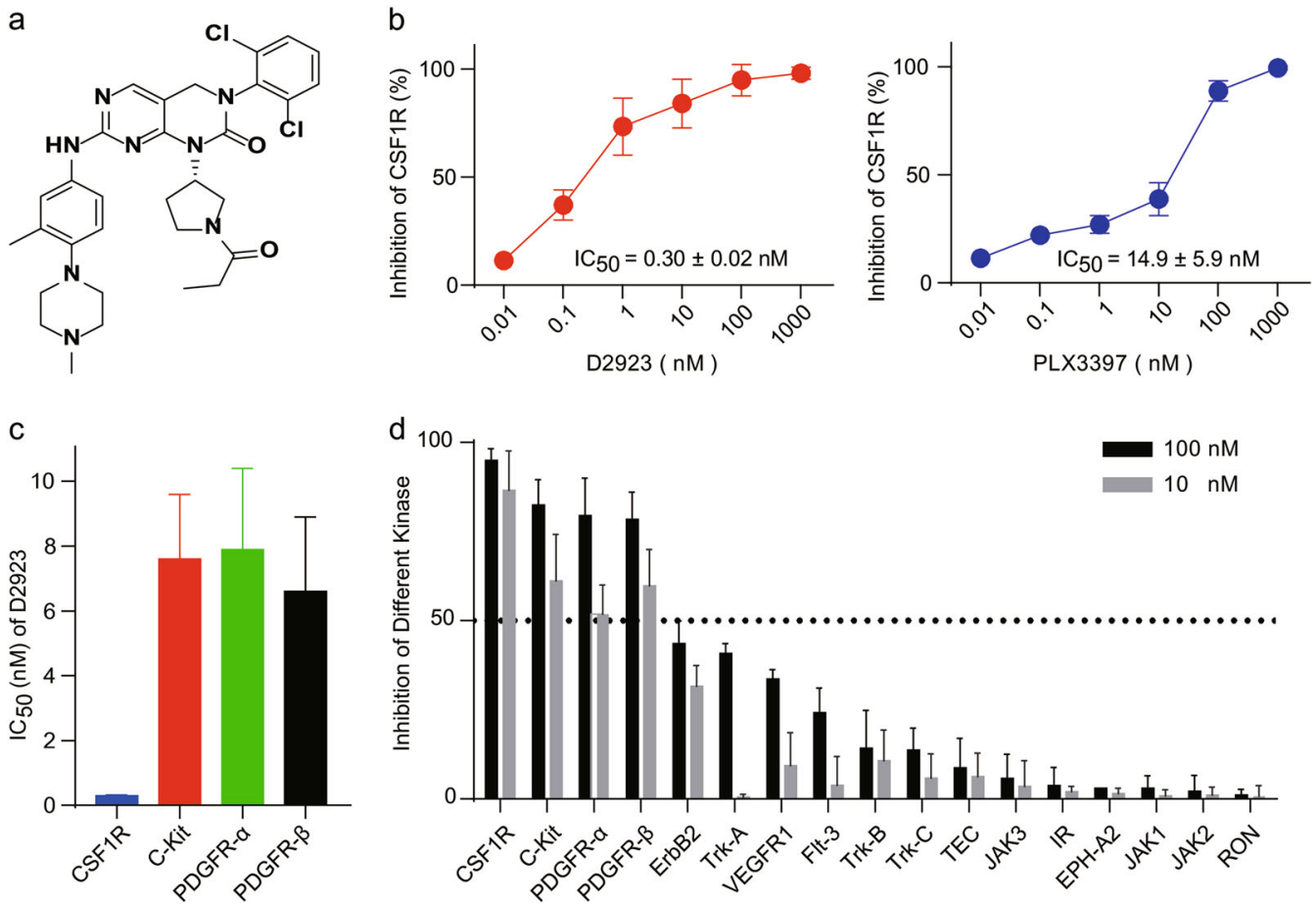

Fig. 1 a The chemical structure of compound D2923. b D2923 and PLX3397 dose-dependently inhibited the kinase activity of CSF1R. The IC 50 values are shown as the mean \pm SD and the estimated values from three independent experiments. c The kinase inhibitory activity of D2923 against c-Kit, PDGFR $\alpha$, and PDGFR $\beta$ compared with that of CSF1R. d The kinase panel screening data of D2923

In vivo antitumor activity

Six male BALB/CA nude mice (cultivated by Shanghai Institute of Medicine, Chinese Academy of Sciences) were housed per cage for group administration. The mice were 5-6 weeks old, and the initial weight was $22 \pm 2 \mathrm{~g}$. The animal experiments were performed according to the institutional ethical guidelines of animal care. The cell lines were obtained from the ATCC. The M-NFS-60 cells were injected (s.c.) into the right flank of each mouse at a density of $5 \times 10^{6}$ in $200 \mu \mathrm{L}$, and the resulting tumors were allowed to grow to $600 \mathrm{~mm}^{3}$, which was defined as a well-developed tumor. The well-developed tumors were cut into $1.5 \mathrm{~mm}^{3}$ fragments and were transplanted (s.c.) into the right flanks of nude mice using a trocar. When the tumor volume reached $200 \mathrm{~mm}^{3}$, the mice were randomly assigned into the control and treatment groups ( $n=6$ per group). The control group was given vehicle alone, and the treatment groups received a $40 \mathrm{mg} / \mathrm{kg}$ oral dose of PLX3397 or 80 and $40 \mathrm{mg} /$ $\mathrm{kg}$ oral doses of D2923 twice daily. The tumor sizes and animal weights were measured twice per week using a microcaliper and weight scale, respectively. The tumor volume $(V$ was calculated as follows: $V=0.5 \times$ [length $(\mathrm{mm}) \times$ width $^{2}\left(\mathrm{~mm}^{2}\right)$ ]. The individual relative tumor volume (RTV) was calculated as follows: $R T V=V_{t} / V_{0}$, where $V_{\mathrm{t}}$ is the volume on each day, and $V_{0}$ represents the volume at the beginning of the treatment. The RTV is shown on indicated days as the mean $\pm S D$ as indicated for the groups of mice.

\section{Immunohistochemistry}

The tumor samples were fixed in formalin for over $24 \mathrm{~h}$, were transferred to $70 \%$ ethanol, and were embedded in paraffin wax. The sections $(5 \mu \mathrm{M})$ were cut and baked onto microscope slides. The slides were incubated with p-CSF1R (\#3155S, Cell Signaling Technologies, Cambridge, MA, USA), F4/80 (MCA497G, Bio-Rad, Cal, USA) and Ki67 (ab16667, Abcam, CSPC, UK). Secondary antibodies were added, and the slides were visualized using a colorimetric method (DAB kit; ZSGB-Bio, Beijing, China). The stained sections were imaged using a BX51 Olympus microscope and a Microfire Digital Camera (Olympus). We adopted the multiplicative quick score method (QS) to assess the expression of different proteins. This system accounts for both the intensity and the extent of cell staining. Briefly, the proportion of positive cells was estimated and given a percentage score on a scale from 1 to $6(1=1 \%-4 \%$; $2=5 \%-19 \% ; 3=20 \%-39 \% ; 4=40 \%-59 \% ; 5=60 \%-79 \%$; and $6=$ $80 \%-100 \%)$. The average intensity of the positively staining cells was given an intensity score from 0 to $3(0=$ no staining; $1=$ weak, $2=$ intermediate, and $3=$ strong staining). The QS was then calculated by multiplying the percentage score by the intensity score to yield a minimum value of 0 and a maximum value of 18 .

\section{RESULTS}

Structure and kinase inhibitory activity of compound D2923 The chemical structure of compound D2923 is shown in Fig. 1a. The effects of D2923 against CSF1R kinase activity was evaluated using an ELISA kinase assay with human recombinant enzymes. D2923 potently and dose-dependently inhibited the kinase activity of CSF1R, with an $\mathrm{IC}_{50}$ value at $0.30 \pm 0.02 \mathrm{nM}$ (Fig. $1 \mathrm{~b}$, left), which is more potent than the control compound PLX3397 $\left(\mathrm{IC}_{50}=\right.$ $14.9 \pm 5.9 \mathrm{nM}$ ) (Fig. 1b, right). To identify other potential targets, the effects of D2923 against CSF1R highly related class III kinases, including c-Kit, Flt3, PDGFRa, and PDGFR 3 , were detected. It displayed tenfold less potency on c-Kit, PDGFRa, and PDGFR $\beta$ compared with CSF1R (Fig. 1c). Moreover, it showed 300-fold less potency on Flt3 compared with CSF1R and exhibited no obvious inhibition on a panel of kinases, including ErbB2, Trk-A, VEGFR-1, Trk-B, Trk-C, TEC, JAK3, IR, EphA2, JAK-2, JAK-3, and EphB2, even at $100 \mathrm{nM}$, a concentration that is 300 -fold higher than the $\mathrm{IC}_{50}$ against CSF1R (Fig. 1d). These results demonstrated that D2923 is a potent and relatively selective inhibitor of CSF1R.

D2923 inhibits cell proliferation of macrophages by blocking CSF1R activation

As CSF1R is required for the proliferation and survival of macrophages, the antiproliferative activities of D2923 against macrophages were then examined. Murine peritoneal macrophage RAW264.7 cells and human peripheral blood monocyte THP-1 cells, which express high 
a

RAW264.7

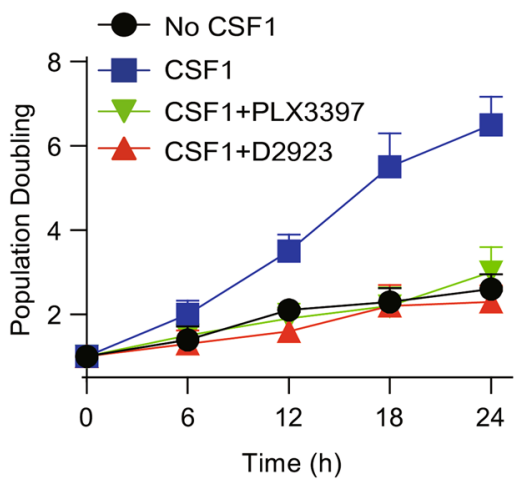

b

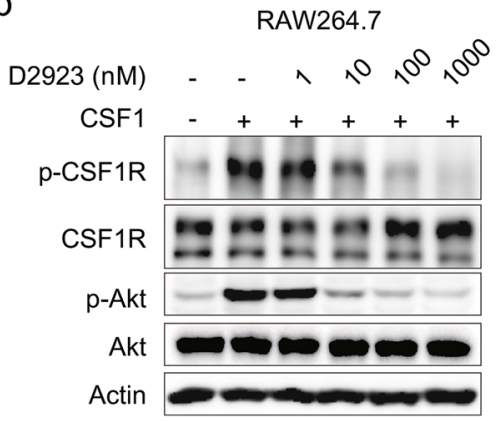

C

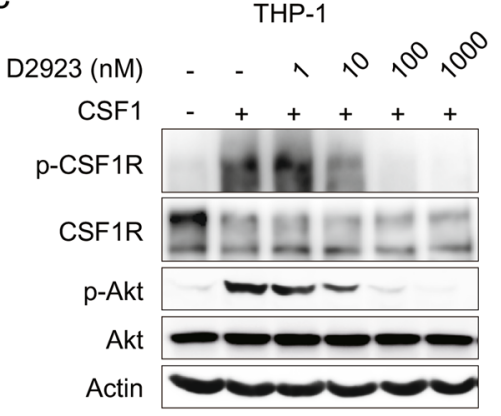

d

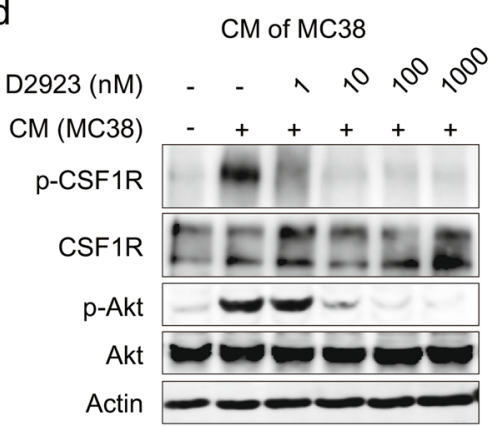

THP-1
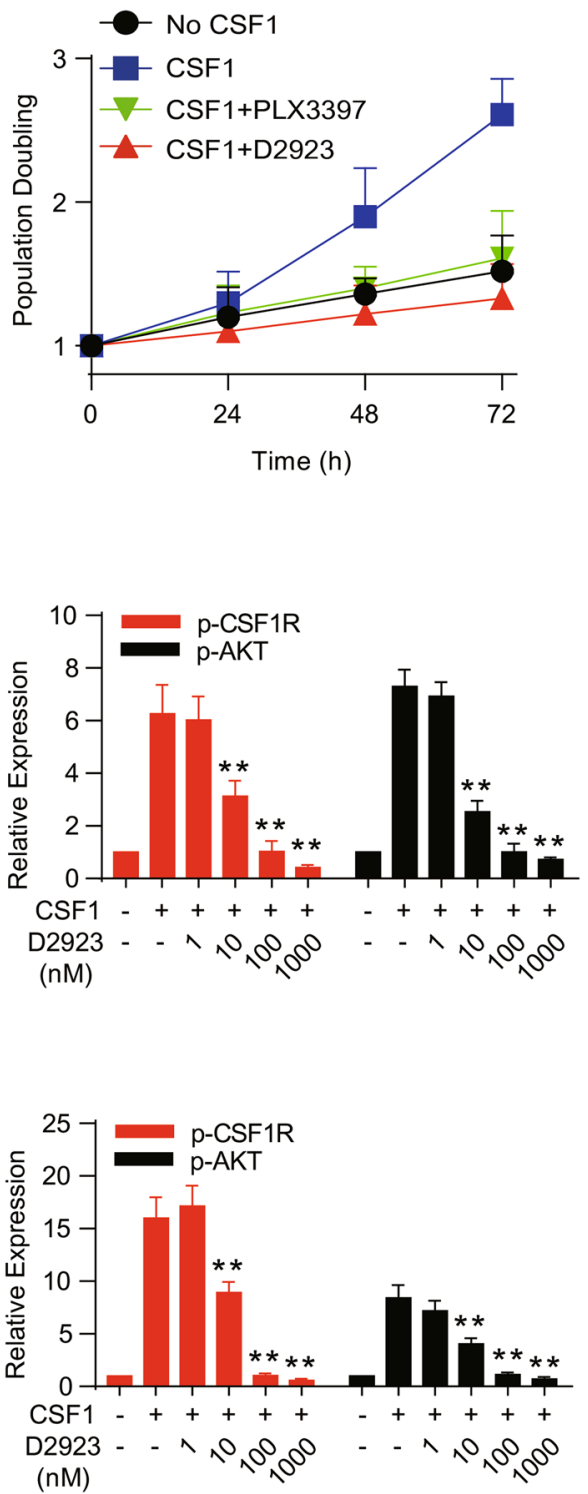

(nM)

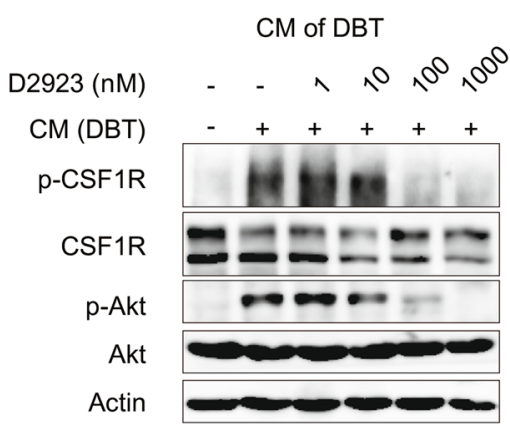

Fig. 2 D2923 blocks the activation of CSF1R in macrophages and suppresses cell proliferation. a D2923 reduced CSF-1-mediated proliferation in mouse peritoneal macrophage RAW264.7 cells and human peripheral blood monocyte THP-1 cells. The concentration of D2923 was $1 \mu \mathrm{M}$ in this test, and PLX3397 (1 $\mu \mathrm{M})$ was used as a positive control. b, c D2923 effectively inhibits the phosphorylation of CSF1R and AKT both in RAW264.7 and THP-1 cells. d D2923 decreased the phosphorylation of CSF1R and AKT in RAW264.7 cells stimulated by the conditional medium (CM) from MC38 or DBT cancer cells ${ }^{* *} P<0.01$ 

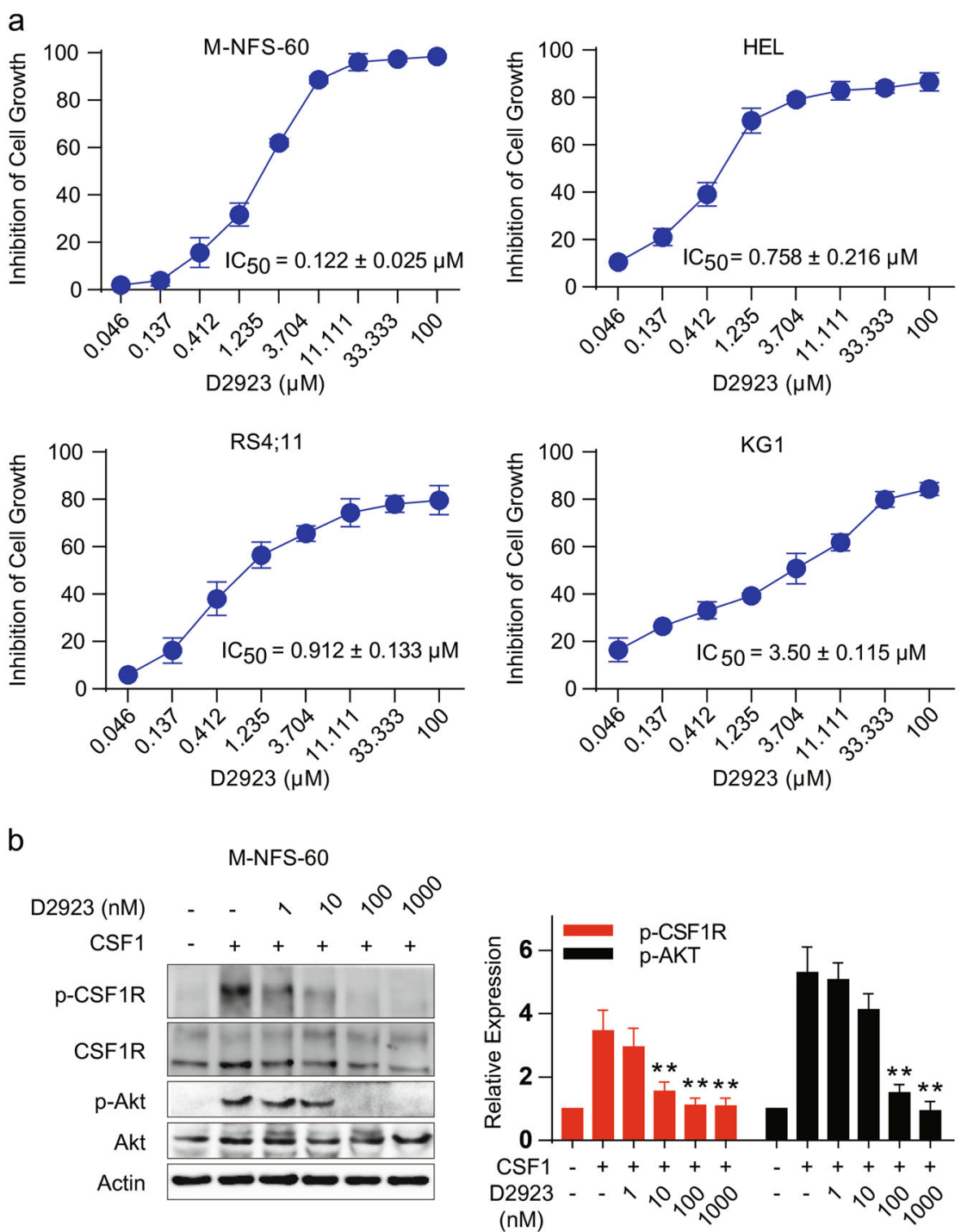

Fig. 3 In vitro antitumor activity of D2923. a D2923 delayed the in vitro growth of a panel of myeloid leukemia cells. b D2923 effectively inhibited CSF-1-stimulated phosphorylation of CSF1R and AKT in M-NFS-60 cells

levels of CSF1R, were used in the test. The addition of extrinsic CSF-1 improved the proliferation of the THP-1 cells and RAW264.7 cells compared with the no CSF-1 control group (Fig. 2a). D2923 treatment at a concentration of $1 \mu \mathrm{M}$ significantly inhibited CSF-1-induced proliferation in these cells, at a similar potency as the reference compound PLX3397 at the same concentration (Fig. 2a).

To evaluate the cellular activity of D2923 against CSF1R, we analyzed its effects on the phosphorylation of CSF1R induced by CSF-1 in macrophages. D2923 significantly inhibited CSF1R phosphorylation in RAW264.7 cells, in a dose-dependent manner (Fig. 2b). A pretty low concentration of D2923, at $10 \mathrm{nM}$, caused $50 \%$ inhibition of CSF1R phosphorylation, and $100 \mathrm{nM}$ of D2923 lead to $80 \%$ inhibition. In accordance with the observation of p-CSF1R inhibition, the phosphorylation of AKT, one of the key molecules downstream of CSF1R, was also significantly decreased (Fig. 2b). Moreover, similar results were also obtained in the THP-1 cells, as evidenced by the potent reduction of $p$-CSF1R and p-AKT expression caused by the D2923 treatment (Fig. 2c).

It is reported that cancer cells secrete superfluous CSF-1. We thus asked whether the conditional medium (CM) from cancer cells could activate CSF1R in macrophages and whether it could be blocked in the presence of D2923. The CM from the mouse cancer cell lines MC38 or DBT were used to stimulate RAW264.7 cells that were starved for $2 \mathrm{~h}$. The result showed that the CM from these tumor cells effectively induced the phosphorylation of CSF1R and AKT in RAW264.7 macrophages and that D2923 treatment efficiently blocked CSF1R activation as well as downstream signaling transduction (Fig. 2d). Collectively, these results demonstrated that D2923 effectively suppressed the phosphorylation of CSF1R and the downstream signaling transduction and, thus, inhibited the proliferation of macrophages.

D2923 delays the proliferation of myelogenous leukemia cells by inhibiting CSF1R

The potency of D2923 against CSF-1-mediated tumor cell proliferation was further assessed in a small panel of myelogenous leukemia cell lines, including M-NFS-60, HEL, RS4;11, and KG1 cells. D2923 markedly inhibited the CSF-1-stimulated proliferation of these cells in a dose-dependent manner, with an $\mathrm{IC}_{50}$ value ranging from 0.122 to $3.5 \mu \mathrm{M}$ (Fig. 3a). The M-NFS-60 cells, which are highly dependent on CSF-1 for survival $[13,29]$, showed the highest sensitivity to D2923 treatment, and thus, the efficacy of D2923 in the M-NFS-60 cells was examined subsequently.

Target inhibition of D2923 in the M-NFS-60 cells was further examined. The western blot results demonstrated that D2923 
a
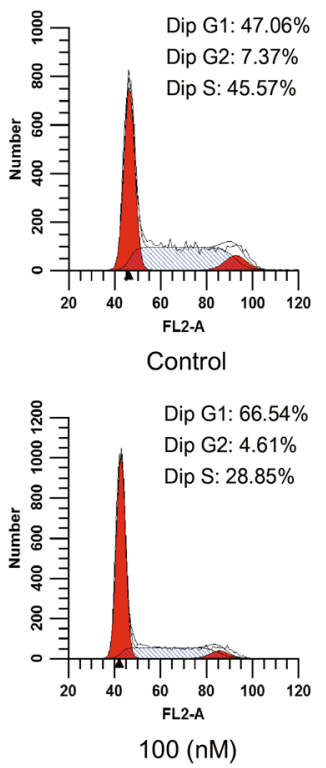

b
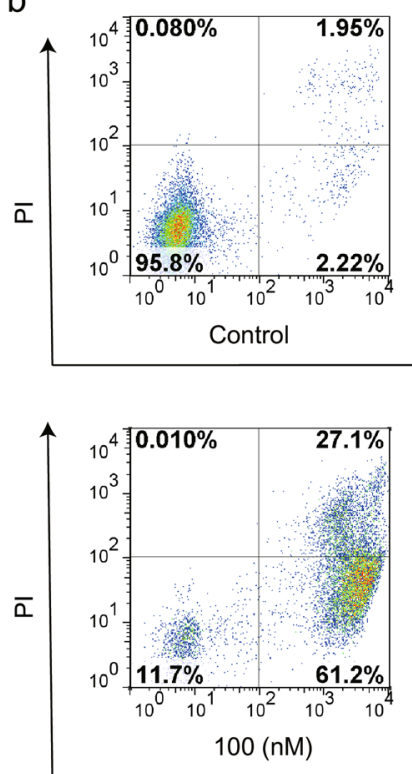
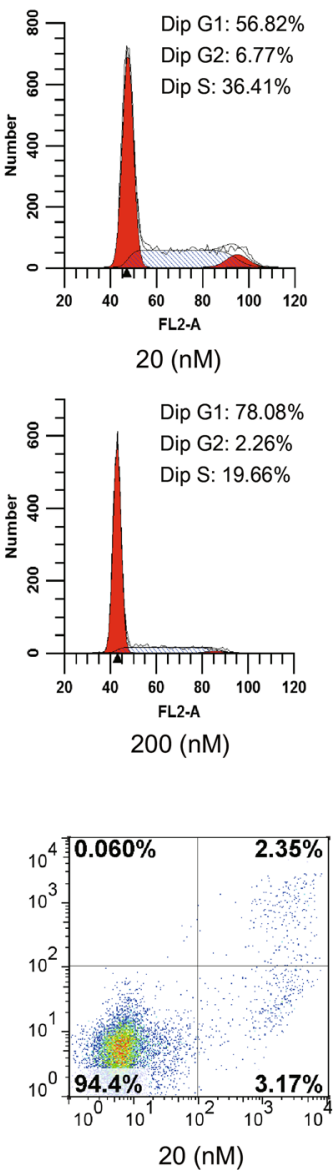

Annexin V
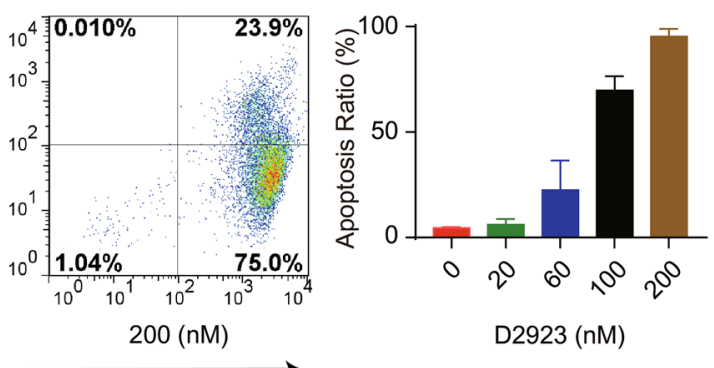

Annexin $\mathrm{V}$

Fig. 4 D2923 induces G1 cell cycle arrest and apoptosis in M-NFS-60 cells. a The effect of D2923 on the M-NFS-60 cell cycle. The cells were treated with the indicated concentrations of D2923 for $48 \mathrm{~h}$. The percentages of cells in the different cell cycle phases were determined by FACS and were analyzed with ModiftLT. b D2923 induced apoptosis in M-NFS-60 cells. The cells were treated with D2923 for 48 h, and the percentages of cells in the different apoptosis phases were determined by FACS and were analyzed with Flowjo VX. Each experiment was conducted independently three times

potently inhibited the activation of CSF1R induced by CSF-1, in a dose-dependent manner. The phosphorylation of CSF1R was completely inhibited at the concentration of $100 \mathrm{nM}$ (Fig. 3b). Concurrently, the phosphorylation of AKT was also potently suppressed by the D2923 treatment (Fig. 3b). Thus, the data demonstrated that D2923 effectively reduced the in vitro proliferation of myelogenous leukemia cells by blocking the activation and the downstream signaling transduction of CSF-1/CSF1R.

D2923 elicits significant effects on G1 cell cycle arrest and apoptosis in M-NFS-60 cells

The effect of D2923 on the cell cycle progression in M-NFS-60 cells was also investigated. After $48 \mathrm{~h}$ of treatment in the M-NFS-60 cells, D2923 significantly arrested cell cycle progression at the G1 phase (Fig. 4a). Exposure to $20 \mathrm{nM}$ of D2923 arrested $56.82 \%$ of the cells occupying the $\mathrm{G} 1$ phase, compared with $47.06 \%$ of the control cells. Approximately $78.08 \%$ of the cells were arrested in the G1 phase when the concentration reached $200 \mathrm{nM}$ (Fig. 4b). We then proceeded to test the effects of D2923 on apoptosis in M-NFS-60 cells. As illustrated in Fig. 4b, D2923 triggers concentration-dependent apoptosis in the cells, with ratios of 15 and $98 \%$ at concentrations of 60 and $200 \mathrm{nM}$, respectively (Fig. 4b), compared with $4 \%$ in the untreated control cells. These results demonstrated that D2923, as a new inhibitor of CSF1R, induced apoptosis and G1 phase cell cycle arrest, which thus suppressed the growth of M-NFS-60 cells. 

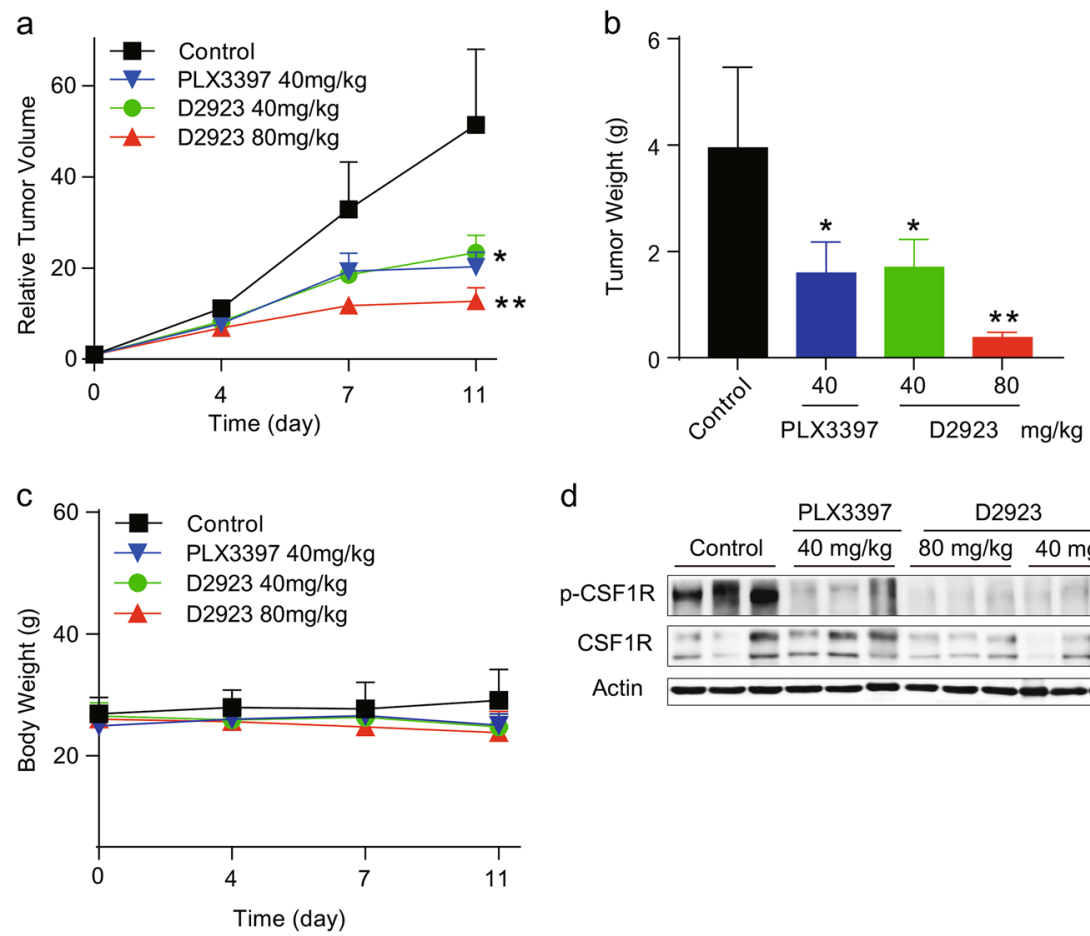

d

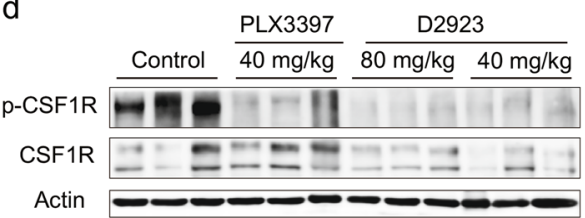

e
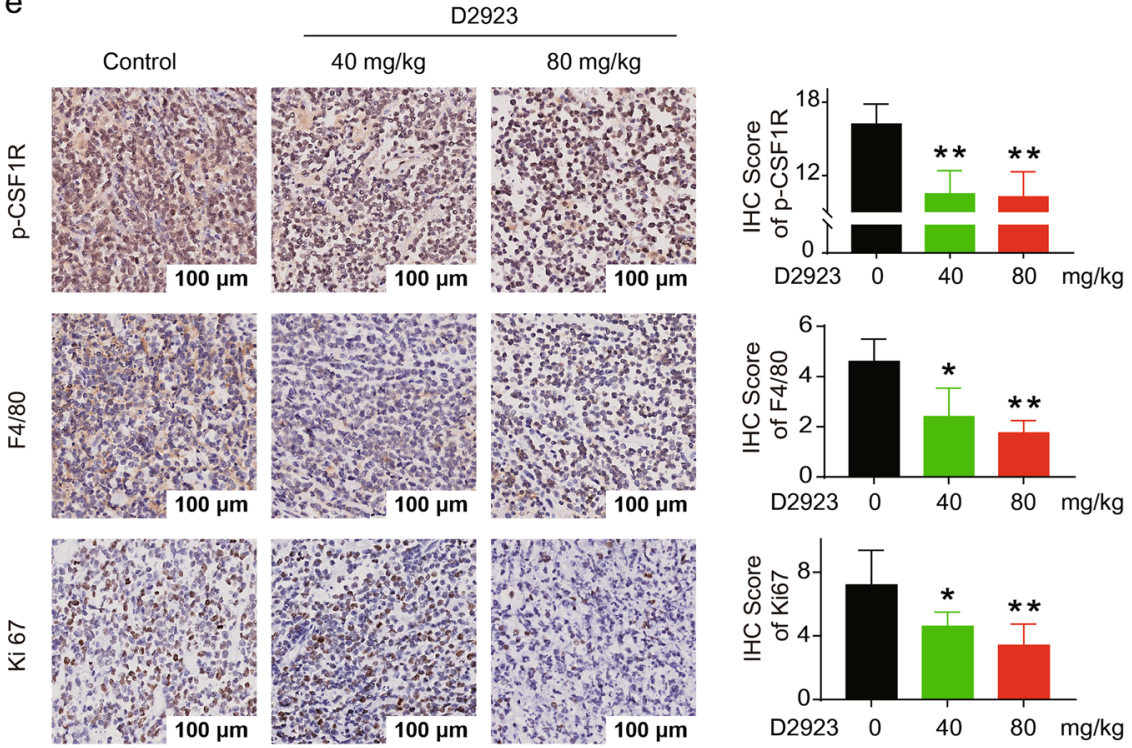

Fig. 5 D2923 exerts antitumor activity in vivo. a D2923 inhibited the growth of M-NFS-60 xenografts. Nude mice bearing with established M-NFS-60 tumors were orally administered, or not, D2923 $(80 \mathrm{mg} / \mathrm{kg}$ or $40 \mathrm{mg} / \mathrm{kg}$, twice daily), using PLX3397 (40 mg/kg, twice daily) as a positive control. The RTVs are expressed as the mean \pm SD. A significant difference from the vehicle group was determined using a $t$ test, ${ }^{*} P<0.05,{ }^{* *} P<0.01$. b The tumor weights were monitored on day 11 . $\mathbf{c}$ The body weight of each mouse was measured during the treatment. d The expression of p-CSF1R, F4/80, and Ki67 in the tumor tissue was examined using a western blot analysis (d) or IHC (e)

D2923 inhibits CSF1R-mediated tumor growth in vivo

To assess the in vivo antitumor efficacy of D2923, the M-NFS-60 xenograft mouse model was well established. D2923 was orally administered twice daily at doses of 40 or $80 \mathrm{mg} / \mathrm{kg}$. The results showed that D2923 suppressed tumor growth in a dosedependent manner, with inhibition rates of 50 and $88 \%$ at doses of 40 and $80 \mathrm{mg} / \mathrm{kg}$ (Fig. 5a), respectively. The endpoint tumor weights in the D2923-treated group were significantly reduced compared with those treated with the vehicle control (Fig. 5b). Notably, D2923 displayed a similar efficacy as PLX3397 in this model when given at the same dose and schedule (Fig. 5a, b).
Additionally, D2923 was well tolerated, with no significant bodyweight loss in the treatment groups (Fig. 5c).

The tumors were then analyzed by a western blot and immunohistochemistry (IHC). The western blot result showed that the phosphorylation of CSF1R in the tumor tissue was markedly inhibited by the D2923 treatment (Fig. 5d). Consistent with this, the results of the IHC confirmed that the phosphorylation of CSF1R was inhibited dramatically (Fig. 5e). The expression of F4/80, a marker of macrophages, was subsequently examined using the IHC method. Intratumoral macrophages were inhibited after oral administration of D2923 (Fig. 5e), clearly indicating that an oral treatment with D2923 effectively suppressed 
macrophage infiltration in xenograft tumors. Moreover, the expression of Ki67, a proliferation marker, was also measured, and the results demonstrated that Ki67 expression was significantly decreased in the 40 and $80 \mathrm{mg} / \mathrm{kg}$ D2923 treatment groups compared with the control group (Fig. 5e), indicating that D2923 possessed potent antiproliferation activity in vivo. These data collectively convinced us that D2923 possessed potent in vivo antitumor activity, mainly by decreasing the number of macrophages in the tumor as well as inhibiting the proliferation of tumor cells.

\section{DISCUSSION}

With the great success of immune checkpoint therapies in recent years, immune cells in the tumor microenvironment have become a focus of cancer research and pharmaceutical development [30-32]. Macrophages represent the most abundant cells of the immune system found within the stroma of some malignancies and, thus, have drawn great attention in cancer therapy. CSF-1 is a key cytokine involved in recruitment and activation of tissue macrophages, exerting these effects by binding to the highaffinity receptor tyrosine kinase CSF1R. The overexpression of CSF1R is closely associated with increased TAMs, the malignance of tumor progress, the promotion of angiogenesis, and resistance to approved therapies [18]. Therefore, inhibiting CSF1R signaling may have a significant potential for the treatment of human cancers by remodeling a favorable tumor microenvironment. Several types of cancers are reported to be highly dependent on CSF-1/CSF1R for growth, such as tenosynovial giant-cell tumors (TGCTs), which represent a rare and locally aggressive neoplasm of the joint or tendon sheath [33]. Therefore, CSF1R becomes an attractive therapeutic target, and a couple of selective CSF1R inhibitors, such as PLX3397 and BLZ945, are in clinical development for the treatment of various types of cancers, including TGCTs. However, none of these selective agents is approved for cancer treatment.

Based on this, the aim of this study was to design and identify CSF1R inhibitors with a novel scaffold. We rationally designed and synthesized a series of 2-Oxo-3, 4-dihydropyrimido[4, 5-d] pyrimidines and evaluated their CSF1R inhibition both in cellfree and cell-based assays. D2923 was one of the representative derivatives, possessing a distinct efficacy in the preliminary screening, and was selected for further investigation. Our data showed that D2923 dose-dependently inhibited CSF1R kinase activity, with an $\mathrm{IC}_{50}$ value at $0.30 \mathrm{nM}$, which was more potent than PLX3397 $\left(I C_{50}=15.9 \mathrm{nM}\right)$ and BLZ945 $\left(I C_{50}=1 \mathrm{nM}\right)$. D2923 showed weak or moderate inhibition against highly related kinases of CSF1R that belong to the class III kinase family, and no obvious inhibition was observed on other kinases tested in this study, even at a much higher concentration of D2923. These results indicated that D2923 is a relatively selective inhibitor of CSF1R that deserves further evaluation.

Since CSF-1/CSF1R signaling is important for the growth of macrophages, the effects of D2923 on the proliferation of human THP-1 macrophages and murine RAW264.7 macrophages were examined, and the results demonstrated that D2923 efficiently inhibited the in vitro proliferation of these cells, with a potency similar to PLX3397. The target inhibition results confirmed that D2923 dose-dependently suppressed the activation of CSF1R as well as downstream signaling transduction. Notably, the phosphorylation of CSF1R in macrophages was also induced by conditioned medium of cancer cells, and this activation was dramatically inhibited by D2923 treatment, indicating that D2923 effectively blocked the reciprocal effects between macrophages and tumor cells.

The effects of D2923 on the tumor cells were also tested in this study. It exhibited a potent antiproliferative efficacy on a panel of myeloid leukemia cells, with M-NFS-60 cells displaying the highest sensitivity. Mechanistic studies revealed that D2923 inhibited the activation of CSF1R as well as signaling transduction in M-NFS-60 cells and, thus, resulted in cell cycle arrest and apoptosis in these cells, in a concentration-dependent manner.

Moreover, the in vivo antitumor activity of D2923 was examined using an M-NFS-60 xenograft mouse model. The oral administration of D2923 effectively suppressed tumor growth, possessing a similar potency as PLX3397, without affecting mouse body weight. Consistent with this, the expression of p-CSF1R in the tumor tissue was dramatically decreased and was accompanied by a decrease in the number of macrophages in the tumor. Consistent with this, D2923 treatment also decreased the expression of Ki67, a proliferation marker of tumor cells. These data clearly indicated that as a novel CSF1R inhibitor, D2923 exhibited antitumor activities in vitro and in vivo by decreasing the recruitment of macrophages into the tumor and by suppressing tumor growth that depended on CSF-1.

In total, these findings provide strong support for further development of D2923 as an antitumor agent. Since CSF1R inhibition is best described in clinical trials for TGCT, further studies of D2923 on TGCT or other CSF-1-dependent tumors should be seriously considered. Moreover, it will also be interesting to examine combination therapies involving D2929 with chemotherapy, radiotherapy and immune checkpoint therapy.

\section{ACKNOWLEDGEMENTS}

This research was partly supported by grants from the "Personalized Medicines, Molecular Signature-based Drug Discovery and Development" and the Strategic Priority Research Program of the Chinese Academy of Sciences (Nos. XDA12020203 and XDA12020209 for HX).

\section{AUTHOR CONTRIBUTIONS}

JD, HX, Y-LL, Y-QL, KD, YC, and M-YG designed the study; Y-QL, Y-NW, L-JT, YL, TZ, $\mathrm{Y}-\mathrm{ZC}, \mathrm{FF}, \mathrm{YS}$, and $\mathrm{Y}-\mathrm{YS}$ performed the research; Q-JX and $\mathrm{X}-\mathrm{YL}$ contributed the compound; Y-QL, L-JT, Y-LL, and HX analyzed the data; and HX and Y-QL wrote the paper.

\section{ADDITIONAL INFORMATION}

Competing interests: We declare that we do not have any commercial or associative interest that represents a conflict of interest in connection with the work submitted.

\section{REFERENCES}

1. Verstraete K, Savvides SN. Extracellular assembly and activation principles of oncogenic class III receptor tyrosine kinases. Nat Rev Cancer. 2012;12: 753-66.

2. Stanley ER, Chitu V. CSF-1 receptor signaling in myeloid cells. Cold Spring Harb Perspect Biol. 2014;6 a021857.

3. Jinushi M, Komohara Y. Tumor-associated macrophages as an emerging target against tumors: creating a new path from bench to bedside. Biochim Biophys Acta. 2015;1855:123-30.

4. Nandi S, Gokhan S, Dai XM, Wei S, Enikolopov G, Lin H, et al. The CSF-1 receptor ligands IL-34 and CSF-1 exhibit distinct developmental brain expression patterns and regulate neural progenitor cell maintenance and maturation. Dev Biol. 2012;367:100-13.

5. Hume DA, MacDonald KP. Therapeutic applications of macrophage colonystimulating factor-1 (CSF-1) and antagonists of CSF-1 receptor (CSF-1R) signaling. Blood. 2012;119:1810-20.

6. Kacinski B. CSF-1 and its receptor in ovarian, endometrial and breast cancer: campaign against ovarian cancer. Ann Med. 1995;27:79-85.

7. Hambardzumyan $\mathrm{D}$, Gutmann $\mathrm{DH}$, Kettenmann $\mathrm{H}$. The role of microglia and macrophages in glioma maintenance and progression. Nat Neurosci. 2016;19:20-7

8. Pyonteck SM, Akkari L, Schuhmacher AJ, Bowman RL, Sevenich L, Quail DF, et al. CSF-1R inhibition alters macrophage polarization and blocks glioma progression. Nat Med. 2013:19:1264-72.

9. Van Overmeire E, Stijlemans B, Heymann F, Keirsse J, Morias Y, Elkrim Y, et al. MCSF and GM-CSF receptor signaling differentially regulate monocyte maturation 
and macrophage polarization in the tumor microenvironment. Cancer Res. 2016;76:35-42.

10. Elmore MR, Najafi AR, Koike MA, Dagher NN, Spangenberg EE, Rice RA, et al. Colony-stimulating factor 1 receptor signaling is necessary for microglia viability, unmasking a microglia progenitor cell in the adult brain. Neuron. 2014;82:380-97.

11. Escamilla J, Schokrpur S, Liu C, Priceman SJ, Moughon D, Jiang Z, et al. CSF1 receptor targeting in prostate cancer reverses macrophage-mediated resistance to androgen blockade therapy. Cancer Res. 2015;75:950-62.

12. Aharinejad S, Abraham D, Paulus $P$, Abri H, Hofmann M, Grossschmidt $K$, et al. Colony-stimulating factor-1 antisense treatment suppresses growth of human tumor xenografts in mice. Cancer Res. 2002;62:5317-24.

13. Conway JG, McDonald B, Parham J, Keith B, Rusnak DW, Shaw E, et al. Inhibition of colony-stimulating-factor-1 signaling in vivo with the orally bioavailable cFMS kinase inhibitor GW2580. Proc Natl Acad Sci USA. 2005;102:16078-83.

14. Coniglio SJ, Eugenin E, Dobrenis K, Stanley ER, West BL, Symons MH, et al. Microglial stimulation of glioblastoma invasion involves epidermal growth factor receptor (EGFR) and colony stimulating factor 1 receptor (CSF-1R) signaling. Mol Med. 2012;18:519-27.

15. Strachan DC, Ruffell B, Oei $Y$, Bissell MJ, Coussens LM, Pryer N, et al. CSF1R inhibition delays cervical and mammary tumor growth in murine models by attenuating the turnover of tumor-associated macrophages and enhancing infiltration by $\mathrm{CD}^{+}$T cells. Oncoimmunology. 2013;2:e26968.

16. Moughon DL, He H, Schokrpur S, Jiang ZK, Yaqoob M, David J, et al. Macrophage blockade using CSF1R inhibitors reverses the vascular leakage underlying malignant ascites in late-stage epithelial ovarian cancer. Cancer Res. 2015;75:4742-52.

17. Von Tresckow B, Morschhauser F, Ribrag V, Topp MS, Chien C, Seetharam S, et al. An open-label, multicenter, phase I/II study of JNJ-40346527, a CSF-1R inhibitor, in patients with relapsed or refractory Hodgkin lymphoma. Clin Cancer Res. 2015;21:1843-50.

18. Ries $\mathrm{CH}$, Hoves $\mathrm{S}$, Cannarile MA, Ruttinger D. CSF-1/CSF-1R targeting agents in clinical development for cancer therapy. Curr Opin Pharmacol. 2015;23:45-51.

19. Garton AJ, Crew AP, Franklin M, Cooke AR, Wynne GM, Castaldo L, et al. OSI-930: a novel selective inhibitor of Kit and kinase insert domain receptor tyrosine kinases with antitumor activity in mouse xenograft models. Cancer Res. 2006;66:1015-24.

20. Liu G, Campbell BT, Holladay MW, Ford Pulido JM, Hua H, Gitnick D, et al. Discovery of AC710, a globally selective inhibitor of platelet-derived growth factor receptor-family kinases. ACS Med Chem Lett. 2012;3:997-1002.

21. Ohno H, Kubo K, Murooka H, Kobayashi $Y$, Nishitoba T, Shibuya M, et al. A c-fms tyrosine kinase inhibitor, Ki20227, suppresses osteoclast differentiation and osteolytic bone destruction in a bone metastasis model. Mol Cancer Ther. 2006:5:2634-43.

22. DeNardo DG, Brennan DJ, Rexhepaj E, Ruffell B, Shiao SL, Madden SF, et al. Leukocyte complexity predicts breast cancer survival and functionally regulates response to chemotherapy. Cancer Discov. 2011;1:54-67.

23. Butowski N, Colman H, De Groot JF, Omuro AM, Nayak L, Wen PY, et al. Orally administered colony stimulating factor 1 receptor inhibitor PLX3397 in recurrent glioblastoma: an Ivy Foundation Early Phase Clinical Trials Consortium phase II study. Neuro Oncol. 2016;18:557-64.

24. Anthony SP, Puzanov PS, Lin KB, Nolop B, West DD. Pharmacodynamicactivity demonstrated in phase I for PLX3397, a selective inhibitor of FMS and Kit. J Clin Oncol. 2011;29:2696.

25. Genovese MC, Hsia E, Belkowski SM, Chien C, Masterson T, Thurmond RL, et al. Results from a Phase IIA Parallel Group Study of JNJ-40346527, an oral CSF-1R inhibitor, in patients with active rheumatoid arthritis despite disease-modifying antirheumatic drug therapy. J Rheumatol. 2015;42:1752.

26. Krauser JA, Jin $Y$, Walles $M$, Pfaar $U$, Sutton $J$, Wiesmann $M$, et al. Phenotypic and metabolic investigation of a CSF-1R kinase receptor inhibitor (BLZ945) and its pharmacologically active metabolite. Xenobiotica. 2015;45: 107-23.

27. Mao Y, Eissler N, Blanc KL, Johnsen Jl, Kogner P, Kiessling R. Targeting suppressive myeloid cells potentiates checkpoint inhibitors to control spontaneous neuroblastoma. Clin Cancer Res. 2016;22:3849-59.

28. Garcia S, Hartkamp LM, Malvar-Fernandez B, van Es IE, Lin $\mathrm{H}$, Wong J, et al. Colony-stimulating factor (CSF) 1 receptor blockade reduces inflammation in human and murine models of rheumatoid arthritis. Arthritis Res Ther. 2016;18:75.

29. Nakoinz I, Lee MT, Weaver JF, Ralph P. Differentiation of the IL-3-dependent NFS60 cell line and adaption to growth in macrophage colony-stimulating factor. J Immunol. 1990;145:860-4.

30. Sharma P, Hu-Lieskovan S, Wargo JA, Ribas A. Primary, adaptive, and acquired resistance to cancer immunotherapy. Cell. 2017;168:707-23.

31. Godfrey DI, Le Nours J, Andrews DM, Uldrich AP, Rossjohn J. Unconventional T cell targets for cancer immunotherapy Immunity. 2018;48:453-73.

32. Andrews MC, Wargo JA. Cancer evolution during immunotherapy. Cell. 2017;171:740-2.

33. Tap WD, Wainberg ZA, Anthony SP, Ibrahim PN, Zhang C, Healey JH, et al. Structure-guided blockade of CSF1R kinase in tenosynovial giant-cell tumor. N Engl J Med. 2015;373:428-37. 